\title{
CFHT ADAPTIVE OPTICS IMAGING OF ACTIVE GALAXIES
}

\author{
J.B. HUTCHINGS \\ National Research Council of Canada \\ Dominion Astrophysical Observatory \\ 5071 W.Saanich Rd, Victoria, B.C., Canada
}

\section{CFHT adaptive optics camera}

The CFHT adaptive optics camera uses a visible light guide signal from a star to operate a bimorph mirror. The system is a unit that is operated by the observer and can be used with CCD or HgCdTe detectors. Pixel sizes are of order $0.04^{\prime \prime}$. The amount of correction varies as the guide star brightness, the angular distance from it, and the natural seeing at the time. With good CFHT conditions, a guide star of 13 mag will give JHK images of FWHM near to the diffraction limit $\left(0.1\right.$ to $\left.0.15^{\prime \prime}\right)$ up to $20^{\prime \prime}$ away. Correction is worse in the optical, but images of $0.2^{\prime \prime}$ or better can be obtained in $\mathrm{R}$ and I-band. The camera performance is described by Rigaut et al (1998).

The camera is being used in programs on active galaxies ranging from the nuclear regions of Seyferts to high redshift QSOs and radio galaxies. Poor weather in 1997 plus delays in a 1K NIR detector have slowed progress. This poster summarizes some programs and results to date. The full details will be published in due course. Other participants in the programs are D. Crampton, S.L. Morris, E. Steinbring, and S. Chapman.

\section{QSOs}

This is a program planned to cover a range of QSO properties and redshifts. The AOB was used to obtain H-band and I-band images of the low luminosity, optically-selected, quasar $1055.3+019$ at $\mathrm{z}=1.06$. The FWHM of the stars were $0.11^{\prime \prime}$ in $\mathrm{H}$ and $0.3^{\prime \prime}$ in I during these observations, and the detectors used had $0.034^{\prime \prime}$ pixels in $\mathrm{H}$ and $0.12^{\prime \prime}$ pixels in $\mathrm{I}$.

The appropriate off-axis point spread functions were measured and modeled. The QSO is clearly resolved in both wavebands, with significant ex- 

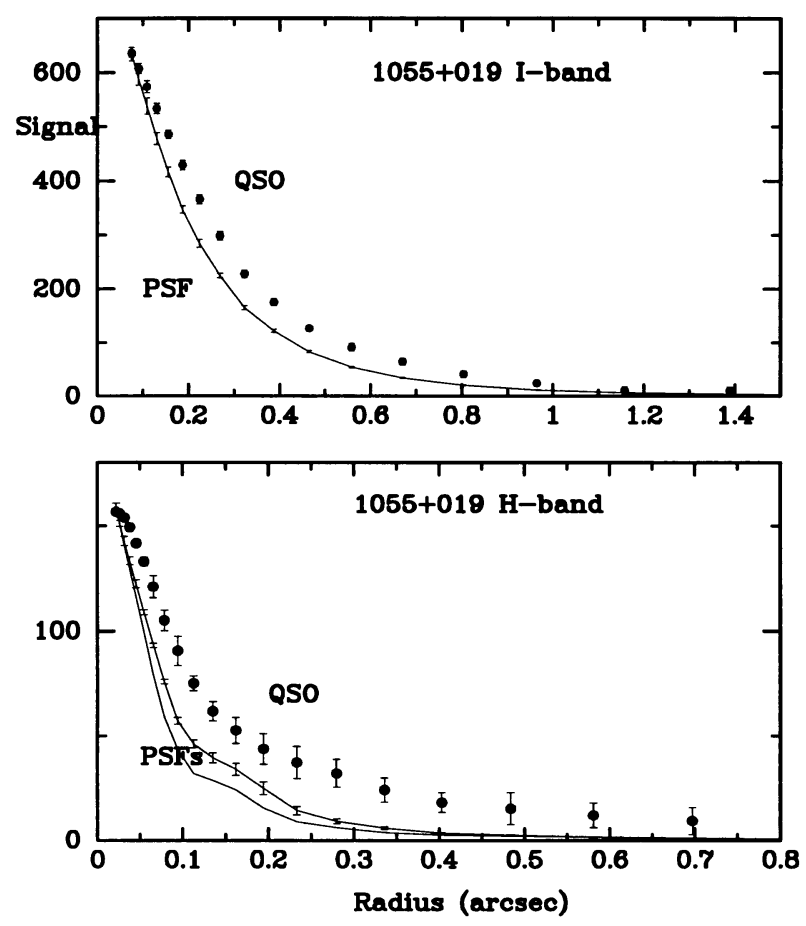

Figure 1. Azimuthally averaged profiles of $z=1.06$ QSO and PSFs in NIR and visible light. H-band PSF shows off-axis correction to QSO.

tended flux within $0.1^{\prime \prime}$ of the nucleus. Overall, it appears to be elongated in the direction of a knot and jet-like feature extending about $1 \operatorname{arcsec} \mathrm{NE}$ of the nucleus visible in the H-band image. There are no other evident close companions, but the host galaxy's complex luminosity profile and offcentered nucleus indicate recent tidal disturbance. The $I-H$ colours of the host galaxy correspond to an unreddened stellar population which is currently star-forming or is within a very short time of ending an initial starburst. The reddening must be small to match any models.

\section{High z Radio sources.}

The AOB is being used for NIR imaging of several radio sources with known optical structure from $H S T$ images. Early results with a $9^{\prime \prime}$ field-of-view, low sensitivity camera were compared with $H S T$ images with similar resolution for two $\mathrm{z} \sim 1.13 \mathrm{C}$ sources. Luminosity profiles and colours were derived from the $\mathrm{J}, \mathrm{H}$ and $\mathrm{K}^{\prime}$ images.

This program will be used to model the stellar populations and other 
continuum light sources in the objects, and compared with the results from the QSO imaging program.

\section{Nuclei of nearby active galaxies.}

Further programs are mapping the inner regions of Seyferts in the NIR to probe the dust that is seen in most objects, to look for structures such as nested bars or features associated with radio jets, to understand the fueling and nuclear processes, and associated circumnuclear star-formation. In these extended and bright structures, it is possible to perform useful deconvolution to improve the images.

Results on the Seyfert galaxy NGC 2992 have been combined with the $H S T$ optical band images with similar resolution. The nuclear region is on the edge of heavy dust obscuration and the NIR image reveals a curved "jet" behind the dust, as well as other knots in the region. The curve corresponds with a radio loop seen in the $6 \mathrm{~cm}$ VLA map. The galaxy has indications of a strong outflow driven by star-formation activity in its inner regions.

\section{References}

Rigaut et al. 1998, PASP, in press 